INPLASY

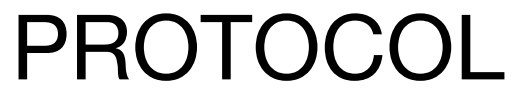

To cite: Shao et al. Efficacy and safety of traditional Chinese patent medicine for Diabetic peripheral neuropathy: A protocol for Bayesian network metaanalysis. Inplasy protocol 202220088. doi:

10.37766/inplasy2022.2.0088

Received: 21 February 2022

Published: 21 February 2022

Corresponding author:

Shichuan Shao

sdstastsq@163.com

Author Affiliation:

Tai'an City Central Hospital

Support: Technology

Development Project.

Review Stage at time of this submission: The review has not yet started.

Conflicts of interest:

None declared.

\section{Efficacy and safety of traditional Chinese patent medicine for Diabetic peripheral neuropathy: A protocol for Bayesian network meta-analysis}

Shao, YZ1; Sun, XN2; Sun, BM33; Shao, SC4.

Review question / Objective: To compare the clinical efficacy and safety of different traditional Chinese patent medicine(TCPM) in the treatment of Diabetic peripheral neuropathy (DPN).

Information sources: Cochrane Library, ClinicalTrials, PubMed, Embase, China National Knowledge Infrastructure Database, Wanfang database, China Science and Technology Journal Database and China Biomedical Literature Database.

INPLASY registration number: This protocol was registered with the International Platform of Registered Systematic Review and Meta-Analysis Protocols (INPLASY) on 21 February 2022 and was last updated on 21 February 2022 (registration number INPLASY202220088).

\section{INTRODUCTION}

Review question / Objective: To compare the clinical efficacy and safety of different traditional Chinese patent medicine(TCPM) in the treatment of Diabetic peripheral neuropathy (DPN).
Condition being studied: With the aging of the population and modern lifestyle changes, the prevalence of diabetes mellitus has increased dramatically. According to the latest report, the people suffering from diabetes were $\mathbf{4 6 3}$ million in 2019 and the number is expected to 700 million in 2045. Diabetic peripheral 
neuropathy (DPN) is the most common complication of diabetic, with a prevalence of $50 \%$ in the diabetes population. Many patients with DPN present symptoms such as limb numbness,pain, dysesthesias, muscle weakness or atrophy. As the disease progresses, the skin of the patient's feet is vulnerable to injury due to unresponsive algesthesia and thermesthesia caused by sensory nerves impairment. More seriously, this condition can lead to foot ulcer and even amputation if not properly cared for. At the same time, these symptoms often have a negative impact on patients' mental state,sleep patterns and autonomic dysfunction. Some studies have reported that DPN, especially painful DPN, are susceptible to depression. The mechanism of DPN has not been fully elucidated yet. Studies have shown that may be related to a variety of factors such as metabolic disorders, microvascular disease, oxidative stress and inflammatory response. Treatment involving Western medicine mainly includes glycemic control,blood pressure control, vasodilation, microcirculation improvement, anti-oxidative stress, neurotrophic action, and pain relief. Although these strategies can temporarily relieve the clinical symptoms, the longterm effect is not very satisfactory. Therefore, the therapeutic strategy for DPN still needs to be further investigated in order to delay the evolution of DPN. With the in-depth research on traditional Chinese medicine(TCM) and the rapid development of TCMpreparation technology, a large number of TCPM are widely used in clinical practice and have been shown to exert precise curative effects through multiple components, multiple targets and multiple pathways.For example,Salvia Miltiorrhiza and Ligustrazine Hydrochloride Injection is shown to improve the median nerve and peroneal nerve conduction velocity through reducing oxidative stress. It has been reported that the combination of TCPM for blood-activating and stasis-removing with Western medicine has complementary advantages for the treatment of DPN, which can reduce the blood flow resistance, promote tissues repair, and improve nerve conduction velocity, thus improving clinical efficacy. However, there is a wide variety of TCPM and research based on evidence-based medical comparing the efficacy of different TCPM is lacking, which makes clinical decision more difficult. Bayesian network metaanalysis provides an efficient solution to this situation. It enables the results of studies to be combined and analyzed. The greatest advantage of Bayesian network meta-analysis is that it allows for precise quantification of different interventions and a comprehensive ranking according to outcome indicators, which in turn facilitates the selection of the optimal treatment strategy. Therefore, in this study, a network meta-analysis was conducted on the TCPM commonly used in clinical practice for the treatment of DPN, in order to provide different clinical decision options to choose from. this study conducted a network meta-analysis of studies using TCPM to treat DPN, in order to provide different programs to choose from.

\section{METHODS}

Participant or population: Patients included in the study were in accordance with the diabetes clinical practice guidelines. No restriction on gender, race, disease duration, etc.

Intervention: The experimental group was treated with TCPM combined with mecobalamin. Any administrated methods such as tablet form and intravenous injection will beincluded. The control group was treated with mecobalamin. The experimental group was treated with TCPM combined with mecobalamin. Any administrated methods such as tablet form and intravenous injection will be included. The control and experimental groups were required to use the same basic interventions, such as blood glucose control, blood pressure control, dietary guidance, and exercise guidance.

Comparator: The control group was treated with mecobalamin. The control and experimental groups were required to use 
the same basic interventions, such as blood glucose control, blood pressure control, dietary guidance, and exercise guidance.

Study designs to be included: Published randomized controlled trials(RCTs).

Eligibility criteria: Inclusion criteria(1)Study type:Published randomized controlled trials(RCTs).(2)Participants: Patients included in the study were in accordance with the diabetes clinical practice guidelines. No restriction on gender, race, disease duration, etc.(3)Interventions:The control group was treated with mecobalamin. The experimental group was treated with TCPM combined with mecobalamin.Any administrated methods such as tablet form and intravenous injection will be included. The control and experimental groups were required to use the same basic interventions, such as blood glucose control, blood pressure control, dietary guidance, and exercise guidance.(4)Outcome indicators:Primary outcome indicators: The motor conduction velocity(MCV) and sensory conduction velocity(SCV) of median nerve and peroneal nerve.Secondary outcome indicators: Clinical symptoms improvement rate, tendon reflexes improvement rate, sensory function improvement rate, side effect incidence.Exclusion criteria(1) Participants were combined with serious diseases, such as diabetic foot, cerebrovascular lesions, etc.; (2) The experimental group used other treatments or drugs that affected the study results, such as acupoint injection, Chinese medicine fumigation, acupuncture,etc.; (3) The data information was incomplete or problematic; (4) Repeatedly published literature.

Information sources: Cochrane Library, ClinicalTrials, PubMed, Embase, China National Knowledge Infrastructure Database, Wanfang database, China Science and Technology Journal Database and China Biomedical Literature Database.

Main outcome(s): The motor conduction velocity(MCV) and sensory conduction
velocity(SCV) of median nerve and peroneal nerve.

Additional outcome(s): Clinical symptoms improvement rate, tendon reflexes improvement rate, sensory function improvement rate,side effect incidence.

Quality assessment / Risk of bias analysis: The quality of literature was evaluated according to the Cachran Risk of Bias tool (Cochrane ROB). The evaluation included the following 7 aspects: randomization methods, allocation concealment, use of blinding for study personnel and subjects, use of blinding for study results, completeness of outcome data, selective reporting of study results, and the presence of other risks of bias. The results of each evaluation were classified as low risk of bias, high risk of bias, and uncertain risk of bias.

Strategy of data synthesis: Run the WinBUGS 1.4.3 program. Three Markov chain Monte Carlo (MCMC) were selected for simulation and the number of iterations was set to 50,000 , with the first 20,000 for annealing and the last 30,000 for sampling. STATA15.0 software was used to map the network relationships between interventions, calculate the surface under the cumulative ranking area (SUCRA), and rank the efficacy of the interventions based on the results.

Subgroup analysis: We will evaluate gender, age, duration of disease, and year of publication for subgroup analysis.

Sensitivity analysis: By excluding a certain literature, the effect on heterogeneity was observed.

\section{Country(ies) involved: China.}

Keywords: diabetic peripheral neuropathy; traditional Chinese patent medicine; network meta-analysis.

Contributions of each author:

Author 1 - Yuze Shao - The author drafted the manuscript. 
Email: 361811065@qq.com

Author 2 - Xiaonan Sun - The author provided statistical expertise.

Email: suninstitute@163.com

Author 3 - Baomin Sun - The author contributed to the development of the selection criteria, and the risk of bias assessment strategy.

Email: stsdstas@163.com

Author 4 - Shichuan Shao - The author read, provided feedback and approved the final manuscript.

Email: sdstastsq@163.com 